\title{
Exploring mechanisms of change in cognitive therapy and interpersonal psychotherapy for adult depression
}

Citation for published version (APA):

Lemmens, L. H. J. M., Galindo-Garre, F., Arntz, A., Peeters, F., Hollon, S. D., DeRubeis, R. J., \& Huibers, M. J. H. (2017). Exploring mechanisms of change in cognitive therapy and interpersonal psychotherapy for adult depression. Behaviour Research and Therapy, 94, 81-92.

https://doi.org/10.1016/j.brat.2017.05.005

Document status and date:

Published: 01/07/2017

DOI:

10.1016/j.brat.2017.05.005

Document Version:

Publisher's PDF, also known as Version of record

Document license:

Taverne

Please check the document version of this publication:

- A submitted manuscript is the version of the article upon submission and before peer-review. There can be important differences between the submitted version and the official published version of record.

People interested in the research are advised to contact the author for the final version of the publication, or visit the DOI to the publisher's website.

- The final author version and the galley proof are versions of the publication after peer review.

- The final published version features the final layout of the paper including the volume, issue and page numbers.

Link to publication

\footnotetext{
General rights rights.

- You may freely distribute the URL identifying the publication in the public portal. please follow below link for the End User Agreement:

www.umlib.nl/taverne-license

Take down policy

If you believe that this document breaches copyright please contact us at:

repository@maastrichtuniversity.nl

providing details and we will investigate your claim.
}

Copyright and moral rights for the publications made accessible in the public portal are retained by the authors and/or other copyright owners and it is a condition of accessing publications that users recognise and abide by the legal requirements associated with these

- Users may download and print one copy of any publication from the public portal for the purpose of private study or research.

- You may not further distribute the material or use it for any profit-making activity or commercial gain

If the publication is distributed under the terms of Article $25 \mathrm{fa}$ of the Dutch Copyright Act, indicated by the "Taverne" license above, 


\title{
Exploring mechanisms of change in cognitive therapy and interpersonal psychotherapy for adult depression
}

\author{
Lotte H.J.M. Lemmens ${ }^{\text {a, }}$, Francisca Galindo-Garre ${ }^{b}$, Arnoud Arntz ${ }^{\mathrm{a}, \mathrm{c}}$, Frenk Peeters ${ }^{\mathrm{d}}$, \\ Steven D. Hollon ${ }^{\mathrm{e}}$, Robert J. DeRubeis ${ }^{\mathrm{f}}$, Marcus J.H. Huibers ${ }^{\mathrm{a}, \mathrm{g}}$ \\ a Department of Clinical Psychological Science, Maastricht University, P.O. Box 616, 6200 MD Maastricht, The Netherlands \\ b EMGO Institute for Health and Care Research, VU University Medical Center, Van der Boechorststraat 7, 1081 BT Amsterdam, The Netherlands

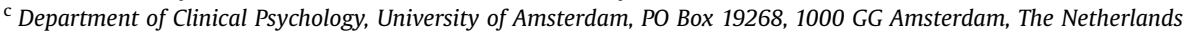 \\ ${ }^{\mathrm{d}}$ Department of Psychiatry and Neuropsychology, Maastricht University, P.O. Box 616, 6200 MD Maastricht, The Netherlands \\ e Department of Psychology, Vanderbilt University, 306 Wilson Hall, Nashville, TN, USA \\ ${ }^{\mathrm{f}}$ Department of Psychology, University of Pennsylvania, 3720 Walnut Street, Philadelphia, PA 19104-6241, USA \\ ${ }^{g}$ Department of Clinical Psychology, VU University Amsterdam, Van der Boechorststraat 1, 1081 BT Amsterdam, The Netherlands
}

\section{A R T I C L E I N F O}

\section{Article history:}

Received 22 April 2016

Received in revised form

3 May 2017

Accepted 6 May 2017

Available online 8 May 2017

\section{Keywords:}

Psychotherapy

Mechanisms of change

Mediators

Cognitive therapy

Interpersonal psychotherapy

Depression

RCT

\begin{abstract}
A B S T R A C T
The present study explored the temporal relationships between change in five candidate causal mechanisms and change in depressive symptoms in a randomized comparison of individual Cognitive Therapy (CT) and Interpersonal Psychotherapy (IPT) for adult depression. Furthermore, hypotheses concerning the mediation of change in these treatments were tested. Patients were 151 depressed adult outpatients treated with either CT $(n=76)$ or IPT $(n=75)$. Depression severity was assessed with the BDI-II. Candidate mediators included both therapy-specific as well as common factors. Measures were taken multiple times over the course of treatment (baseline, mid-, and post-treatment). Pearson's correlations and Latent-Difference-Score models were used to examine the direct and indirect relationships between (change in) the candidate mediators and (subsequent) (change in) depression. Patients showed improvement on all measures. No differential effects in pre- to post-treatment changes were observed between the two conditions. However, change in interpersonal functioning occurred more rapidly in IPT. Only little empirical support for the respective theoretical models of change in CT and IPT was found. Future studies should pay special attention to the timing of assessments and within-patient variance.
\end{abstract} (C) 2017 Elsevier Ltd. All rights reserved.

\section{Introduction}

The efficacy of Cognitive Therapy (CT) and Interpersonal Psychotherapy (IPT) for the treatment of Major Depressive Disorder (MDD) has been well established (Cuijpers et al., 2011; Cuijpers, van Straten, Andersson, \& van Oppen, 2008; Hollon \& Ponniah, 2010; Hollon, Thase, \& Markowitz, 2002). Despite this proven efficacy, research has provided relatively little evidence that speaks to the mechanisms through which CT and IPT lead to symptom change, and whether they do so for reasons hypothesized in their respective theories. The psychological processes that are assumed to be responsible for therapeutic change can be represented in research by measures that are proposed to represent mediators (Kazdin,

\footnotetext{
* Corresponding author.

E-mail address: Lotte.Lemmens@Maastrichtuniversity.nl (L.H.J.M. Lemmens).
}

2007). A mediator is a variable that (statistically) explains why and in what way a treatment has an effect on outcome, and can be seen as representing a potential mechanism (the actual process) through which therapeutic change is brought about (Baron \& Kenny, 1986; Kraemer, Wilson, Fairburn, \& Agras, 2002; MacKinnon, Fairchild, \& Fritz, 2007).

It should be noted that statistical mediation is evidence for, but not proof of, the presence or absence of a mechanism. Various other requirements need to be met as well (e.g., Kazdin, 2007; 2009). Probably the most important addition to statistical mediation is demonstrating the direction of causality: to demonstrate that the treatment causes the mediator to change, which in turn causes change in the outcome, and not the other way around (reversed causality). In order to determine the direction of causality, it is important that both the candidate mediator and outcome are assessed at multiple time-points during treatment. 


\subsection{Theorized mechanisms of CT and IPT}

According to their respective theoretical models, different mechanisms are involved in CT and IPT. Cognitive theory states that depression is caused and maintained by dysfunctional beliefs and maladaptive information processing strategies (Beck, 1964). The inner life of depressed patients is said to be dominated by a set of negative (often unrealistic) assumptions about the self, the world and the future. These assumptions are further organized into large knowledge structures called schemas that influence the content and process of thinking in response to stressful life circumstances, thereby maintaining symptoms (Beck, 1987; Clark, Beck, \& Alford, 1999). According to cognitive theory, depression severity can be reduced by altering the function, content and structure of cognitions and schemas associated with negative affect (Beck, Rush, Shaw, \& Emery, 1979). CT therefore focuses on identification and modification of distorted thinking patterns associated with depressed mood. IPT, developed by Klerman, Weissman, Rounsaville, and Chevron (1984), is based on the idea that depression occurs in the context of social and interpersonal events. Once depressed, symptoms of the disorder further compromise interpersonal functioning, causing a downward spiral. IPT tries to understand the social and interpersonal context in which the depressive symptoms arose, and how they relate to the current social and personal context. The theorized mechanism of IPT is that as patients solve their interpersonal problems or their emotions in relation to their problems, the depression will resolve as well (Markowitz \& Weissman, 2004).

The idea that cognitive change accounts for therapeutic change is a popular hypothesis that has motivated dozens of investigations of the role of cognitive change in CT and other treatments for depression (see reviews of e.g., Garrat, Ingram, Rand, \& Sawalani, 2007; Longmore \& Worrell, 2007; Whisman, 1993 for an overview). Unfortunately, the majority of the relevant studies do not address the aspect of temporality. As a result, it remains unclear whether changes in cognitions precede or follow from changes in depression during treatment. Only a few studies of the mechanisms of change in psychotherapy have included efforts to detect the direction of causality (e.g., DeRubeis et al., 1990; Kuyken et al., 2010; Strunk, Brotman, \& DeRubeis, 2010; Warmerdam, van Straten, Jongsma, Twisk, \& Cuijpers, 2010). In a recent analysis of the relevant literature, Lorenzo-Luaces, German, and DeRubeis (2014) argue that the existing research provides some support for the cognitive mediational model, but that the support is not strong. They conclude that insofar as cognitive change is a mechanism, it is likely not specific to $\mathrm{CT}$.

Few investigations of the processes of change in IPT have been published. In 2013, Toth et al. examined mediators of sustained treatment effects of IPT in a sample of economically disadvantaged mothers with MDD. They found that changes in perceived stress and social support mediated treatment outcome eight months after treatment termination. However, no studies so far have examined whether changes in interpersonal functioning during the acute phase of treatment for depression mediate outcomes. Nonetheless, in several studies, the relation between changes in various theorized processes of IPT have been found to correlate with outcome. More specifically, Bernecker (2012) concluded in her summary of this literature that reduction or resolution of interpersonal problems, reduction of attachment-anxiety and avoidance, and improved marital adjustment were associated with treatment outcome. Although informative, these findings do not speak to mechanisms, since the studies were unable to differentiate between cause and effect. As a result, alternative explanations for the relation between theorized processes of IPT and outcome cannot be ruled out. For example, it may well be that IPT leads to initial symptom relief which, in turn, causes the patient to reach out to family and friends, hereby improving interpersonal functioning. In addition, a more recent study by the same group found that changes in interpersonal functioning to were unrelated to outcome (Bernecker, Constantino, Pazzaglia, Ravitz, \& McBride, 2014).

\subsection{Common factors}

Contrary to the view that treatments exert beneficial effects through their own (specific) theorized mechanisms is the idea that treatments work through common factors. One of the most frequently investigated common factor is the therapeutic alliance (Castonguay, Constantino, \& Holtforth, 2006). Therapeutic alliance refers to the collaborative and affective bond between patient and therapist (e.g., Bordin, 1979). It is believed that a strong alliance in which patient and therapist agree on the goals and tasks of the therapy, and feel safe, secure and understood, is associated with change in depressive symptomatology. The (development of the) quality of the alliance throughout treatment is seen to facilitate symptom change. Research has consistently found that a good alliance is indeed associated with better treatment outcomes in various types of psychological treatment, including CT and IPT (see e.g., Horvath, Del Re, Flückiger, \& Symonds, 2011). However, it is premature to conclude that the quality of the alliance plays a causal role. Effect sizes reported in the literature have been relatively small (overall relation of $r=0.28$ in the most recent meta-analysis by Horvath et al., 2011), and - similar to studies examining the specific mechanisms of CT and IPT - few studies have controlled for temporal confounds (Barber, 2009). For both CT and IPT, studies in which the temporal priority has been accounted for have produced mixed results. Some studies have found support for the notion that the quality of (early) alliance does facilitate subsequent symptom change (e.g., Webb et al., 2011; Zuroff \& Blatt, 2006), whereas others have not (e.g., Constantino et al., 2017; DeRubeis \& Feeley, 1990; Feeley, DeRubeis, \& Gelfand, 1999; Gaston, Marmar, Gallagher, \& Thompson, 1991; Strunk et al., 2010). Future research should therefore further examine the causal influence of alliance on symptom change in both interventions. In addition, other common factors have so far been largely overlooked as potential mechanisms of change.

\subsection{Challenges in the field of mechanism research}

Apart from the issue of temporality and conflicting hypotheses about which candidate mediators should be investigated, progress in research on therapeutic mechanisms has been impeded by small sample sizes, and little consensus concerning the statistical approaches and specific methods to be used in tests of mediation (see Lemmens, Müller, Arntz, and Huibers (2016) for more information). Furthermore, because many of the studies have lacked a comparison group, they have not allowed for a direct comparison of mediation patterns between interventions. As a result, there is a need for additional studies with designs suitable for mechanism research. More specifically, RCT's are needed that examine the mediational role of multiple specific- and non-specific candidate mediators, in large, longitudinal studies using up-to-date statistical analyses techniques.

Thus far, there is only one randomized comparison of CT and IPT in which mediation has been a focus (Quilty, McBride, \& Bagby, 2008). Quilty and colleagues examined evidence for the cognitive mediational model and found that CT produced greater change in dysfunctional attitudes than did IPT. Furthermore, they concluded that change in dysfunctional attitudes mediated the effect of CT on depression. However, given their pre-to post-treatment design they could not address the issue of temporal precedence. In addition, 
Quilty et al. focused only on cognitive change. Consequently, the role of interpersonal variables and common factors in a randomized comparison of CT and IPT remains to be studied.

\subsection{Current study}

We recently conducted an RCT investigating the clinical effects of individual CT and IPT for the treatment of adult depression in which we found no significant differences on outcomes in the acute phase (Lemmens et al., 2011, 2015). We now examine the role of five candidate mediators of CT and IPT - specific and non-specific over the course of treatment. A common misconception in the field of mechanism research, dating to the original article by Baron and Kenny (1986), is that it is impossible to perform mediation analyses if group differences are absent. However, as outlined by e.g., Hayes (2009), Kraemer et al. (2002) and MacKinnon et al. (2007), differential treatment effects are not required to establish statistical mediation, as long as there is an interaction between treatment and the mediator. We included a measure of dysfunctional attitudes to represent the cognitive theory and a measure of interpersonal problems to represent interpersonal theory. The working alliance was included as a potential common factor, as were rumination and self-esteem - two other processes that have previously been associated with (change in) depressive symptoms (see Fig. 1). ${ }^{1}$ Candidate mediators and outcome were assessed multiple times over the course of therapy. This temporal design provided the opportunity to examine whether change in proposed mediators preceded, followed from, or went together with changes in depression severity, hereby expanding on prior research on mechanisms. We examined whether scores on the various candidate mediator measures changed over the course of therapy and whether changes on these measures distinguished the two conditions. Furthermore, for each candidate mediator we examined its relation with change in depressive symptoms, both contemporaneously and as a predictor of subsequent change in the outcome variable. To conclude, we investigated whether the theorized processes mediated the relation between treatment and outcome.

We hypothesized that scores on the candidate mediators measures would each, on average, change in an adaptive direction over the course of treatment (i.e., increases in self-esteem and working alliance, and decreases in dysfunctional attitudes, interpersonal problems and rumination). In keeping with the respective theories, we expected the effects of CT to be accounted for by cognitive change (Beck et al., 1979), and the effects of IPT by change in interpersonal functioning (Klerman et al., 1984). More specifically, we expected that $\mathrm{CT}$ would produce greater change in dysfunctional attitudes than in IPT, and that change would predict subsequent reduction of depressive symptomatology in $\mathrm{CT}$, but to a lesser extent in IPT, whereas IPT would produce more change in interpersonal problems and that change would be more associated with depression reduction in IPT than in CT. Common factors were expected to have a comparable impact in both conditions. However, since previous research (e.g., Bernecker et al., 2014; Quilty et al., 2008; Warmerdam et al., 2010) suggests that change in theorized processes may not be modality-specific, we did not rule out the possibility that all candidate mediators would play a role in both interventions (Hollon, DeRubeis, \& Evans, 1987).

\footnotetext{
1 Readers familiar with our design paper (Lemmens et al., 2011) will recognize that our study also included measures of attributional style and cognitive reactivity. However, we did not include them in the current analyses, since these variables were included in the study because of their potential connection to relapse, and not necessarily as mediators of change in the acute phase. Findings on these measures will be presented in a subsequent paper on long-term outcomes.
}

\section{Methods}

\subsection{Design and participants}

Data were collected as part of a large RCT examining the clinical effects and mechanisms of change of individual CT and IPT for depression (Lemmens et al., 2011, 2015). A total of 182 depressed outpatients were randomly allocated to CT $(n=76)$, IPT $(n=75)$, or a 2-month Waiting-List Control condition followed by treatment of choice (WLC; $n=31$ ). For the purpose of the present study, we focused only on the two active conditions and on data collected in the acute phase (baseline to 7 months). Details concerning the design of the study, participants, interventions, and outcomes are reported elsewhere (Lemmens et al., 2011, 2015), and will therefore only be briefly summarized here.

Participants were adult outpatients (18-65 years) referred to the mood disorder program of the Academic Community Mental Health Centre Maastricht (the Netherlands) with a primary diagnosis of MDD confirmed by the Dutch version of the Structured Clinical Interview for DSM-IV Axis I Disorders (SCID-I; First, Spitzer, Gibbon, \& Williams, 1997). Further inclusion criteria were: internet access, an e-mail address and sufficient knowledge of the Dutch language. Patients receiving concomitant pharmacological or psychological treatment at baseline were excluded from the study, as were those at imminent risk for suicide. Other exclusion criteria were: bipolar or chronic depression (current episode $>5$ years); substance abuse/dependence or mental retardation (IQ $<80$ ). The CT protocol followed the guidelines laid out by Beck et al. (1979). The IPT protocol was based on the manual by Klerman et al. (1984). Treatment consisted of individual 45-min sessions. Sessions were offered weekly, but the protocol allowed flexibility in scheduling appointments less frequently, hereby reflecting everyday mental health-care in the Netherlands. All participants provided their informed consent before entering the study. The study was approved by Maastricht University's Ethical Board, and was conducted in accord with relevant ethical guidelines. The study is registered at the Netherlands Trial Register, part of the Dutch Cochrane Centre (ISRCTN67561918).

Both treatments led to considerable improvement in depression severity (pre-post effect size $d=1.72$ in the pooled active conditions) that was sustained up to 5-months follow-up. Response to therapy exceeded response in the WLC condition. No differential effects between treatments were found. Independent assessors rated quality of therapy as being "(very) good" to "excellent" in both conditions. An extensive integrity check differentiated CT and IPT and indicated that therapist adhered to protocol (Lemmens et al., 2015).

\subsection{Outcome measure}

\subsubsection{Depressive symptoms}

The primary outcome was depression severity as measured with the Beck Depression Inventory-II (BDI-II; Beck, Steer, \& Brown, 1996; Dutch translation by van der Does, 2002). The BDI-II is a 21 item self-report questionnaire with items rated on a 4-point Likertscale (0-3). Higher scores indicate higher levels of depression severity (range is 0-63). The instrument has strong psychometric properties as a screening measure for depressive symptom severity (Beck et al., 1996; van der Does, 2002).

\subsection{Potential mediator variables}

\subsubsection{Dysfunctional beliefs}

The presence and intensity of dysfunctional beliefs - a core concept of Beck's cognitive theory - was assessed with the 17-item 


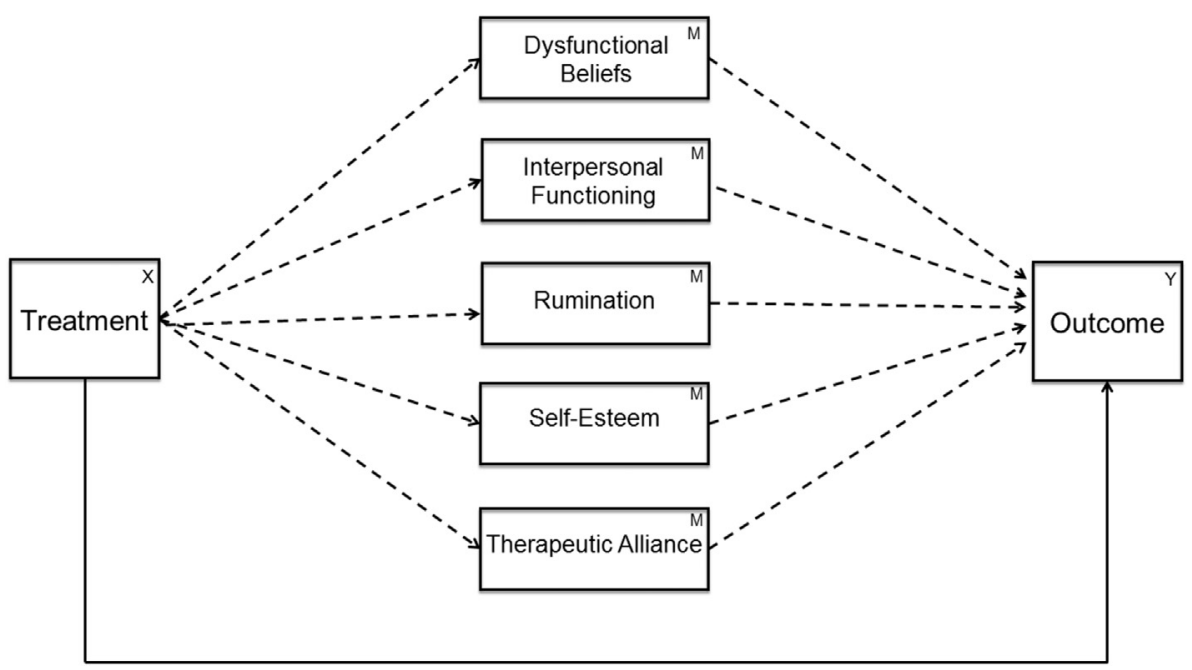

Fig. 1. Theorized and estimated models of direct and indirect effects of psychotherapy on depression severity through five potential mediators. Note: $\mathrm{M}=$ Potential mediator.

Dysfunctional Attitudes Scale (form A) Revised (DAS-A17; de Graaf, Roelofs \& Huibers, 2009). In this shortened version of the original DAS-A by Weissman and Beck (1978), respondents rate the extent to which they agree with a series of dysfunctional assumptions. Items are rated on a 7 -point Likert-scale $(1=$ fully disagree to 7 = fully agree). Higher scores reflect more negative and absolute thinking (range is 17-119). The DAS-A17 is a valid measure of dysfunctional cognitions in depressed patients (de Graaf et al., 2009).

\subsubsection{Interpersonal functioning}

Interpersonal Functioning was assessed with the 64-item version of the Inventory of Interpersonal Problems (IIP-64, henceforth IIP, Horowitz, Alden, Wiggins, \& Pincus, 2000). The IIP is a selfreport measure that assesses the problems that people experience in their relationships with others, and the level of distress associated with this. Items are rated on a 5-point Likert-scale (0-4) with higher scores indicating more interpersonal problems (range is 0-256). The IIP has been shown to have good psychometric properties (Horowitz, Rosenberg, Baer, Ureño, \& Villaseñor, 1988, 2000).

\subsubsection{Rumination}

The Dutch version of the Ruminative Response Scale (RRS-NL; Raes, Hermans, \& Eelen, 2003), originally developed by NolenHoeksema and Morrow (1991), was used to obtain a measure of rumination. The RRS-NL includes 22 items describing responses to depressed mood that are focused on the self, on symptoms, and on possible causes and consequences of mood. Participants read each item and indicate how often they think or do this when they feel sad or depressed. Items are rated on a four point Likert-scale ranging from almost never (1) to almost always (4). The total score is the sum of the 22 items (range is 22-88). The RRS-NL shows good reliability and satisfactory validity (Raes et al., 2003).

\subsubsection{Self-esteem}

The Self-Liking and Self-Competence Scale Revised (SLCS-R; Tafarodi \& Swann, 2001; Dutch version by Vandromme, Hermans, Spruyt, \& Eelen, 2007) is a self-report measure of selfcompetence and self-liking, two dimensions of self-esteem. The SLSC-R contains eight items each rated on a 5-point Likert scale, for each of the two dimensions. Subscale scores can range from 8 to 40 , with higher scores indicating higher self-competence or higher self-liking. A total score is calculated by summing the score of the two subscales. Psychometric properties of the SLSC-R are described as good (Vandromme et al., 2007).

\subsubsection{Therapeutic alliance}

The observer rated version of the Working Alliance Inventory Short (WAI-O-S; Tichenor \& Hill, 1989; Tracey \& Kokotovic, 1989) was used to obtain a measure of the quality of the therapeutic alliance. The WAI-O-S is based on Bordin's (1979) conceptualization of working alliance which states that a strong alliance forms if a therapist and client a) agree on the goals of therapy, b) agree on the tasks needed to meet those goals, and c) have a bond between them that will facilitate this process. Each of the 12 items of the scale (4 for each subscale) are rated on a 7 -point scale ( $1=$ never, to 7 = always), and a higher score indicates a stronger alliance. A total score is obtained by summing up all item scores (range is 12-84). Psychometric properties of the WAI-O-S have been found to be good (Andrusyna, Luborsky, Pham, \& Tang, 2006; Horvath, 1994; Strunk et al., 2010).

\subsection{Recruitment and procedure}

Self-report measures (BDI-II, DAS-A17, IIP, RRS, SLSC-R) were administered at baseline ( 0 months), mid-treatment (3 months) and post-treatment ( 7 months). Our intent in spacing out the assessments to this extent was to examine temporal relations while minimizing the burden for participants. Baseline and posttreatment assessments were administered at the university. Midtreatment assessment took place via the internet. Of the 151 patients that were included in this study, 3 patients $(2 \%)$ did not complete the 3-month assessment. At seven months, data from 14 participants (9\%) were missing. The average number of sessions attended prior to the 3-month assessment was 6.6 $(\mathrm{SD}=2.2)$ for CT and 6.7 ( $\mathrm{SD}=2.7$ ) for IPT. At the 7-month assessment this was 14.0 $(\mathrm{SD}=4.1)$ and $13.8(\mathrm{SD}=4.4)$ for $\mathrm{CT}$ and IPT respectively.

All therapy sessions were videotaped. A selection of these tapes (three per patient) were watched by independent raters and rated on the quality of the therapeutic alliance. Raters were five clinical psychology master students from Maastricht University (the Netherlands), and four experts in the field of CT and IPT. Prior to the study, raters studied relevant literature. In addition, students received approximately 14 hours $(7 \times 2)$ of group training aimed at getting familiar with the treatment models, the concept of working alliance and the rating instrument. The professionals, who were 
already familiar with the treatments and the working alliance, received 4 hours of additional training in how to use the rating instrument. All raters received a detailed instruction manual. All identifying information was removed from the tapes to ensure that raters were blind to session number, treatment outcome and other patient characteristics. Due to the specific therapeutic interventions and the visual character of the study it was not possible to blind raters for treatment allocation and therapist. Ratings were made independently after watching an entire session.

In order to obtain a measure of alliance comparable to the fixed assessment points of the self-report data, we selected therapy sessions that were closest to the assessment points at baseline, 3 and 7 months. Of the 453 sessions $\left(3^{*} 151\right)$ that were selected, 192 tapes were unavailable. This was either because patients did not give permission for videotaping their sessions $(n=34$, resulting in 102 missing tapes), or because recordings of specific sessions were missing or damaged $(n=90)$. As a result, 261 tapes were available for analyses ( $n=88$ at baseline, $n=97$ at 3 months, and $n=76$ at 7 months). There were no significant differences at baseline in sociodemographic variables (gender, age, level of education, work- and marital status) and depression severity between patients who did $v s$. did not give consent for videotaping their sessions, and between patients with complete $v s$. incomplete data. Furthermore, in both comparisons, no significant differences were found with regard to overall change in depression over the course of treatment (BDI-II change from month 0 to month 7). Other details about the recruitment procedure as well as the flow diagram for the study are given elsewhere (Lemmens et al., 2011, 2015).

\subsection{Data analysis}

Data were analysed according to the intention-to-treat principle. Descriptive statistics and correlation analyses were carried out using SPSS version 24 for Windows. Longitudinal Mediation analyses were carried out in M-Plus version 7.1. A full information maximum likelihood estimation algorithm was used for the analyses. Because this algorithm uses all available information under the assumption that the incomplete data are missing at random, missing values were not imputed.

Normality and variability of the data distributions were checked by skewness and kurtosis statistics, histograms and scatter-plots. For each measure, baseline reliability was assessed using Cronbach's alpha $(\alpha)$. Mean scores (SD) at each time-point were determined, and group differences were investigated using independent samples $t$-tests. Pre- to post-treatment effect sizes were calculated to examine the extent to which scores on the various measures changed over the course of treatment. Within-condition change was defined as Cohen's $d=$ (pre-treatment mean - post-treatment mean $) /(\sqrt{ }$ baseline variance $)$. Between-group effect sizes were determined by calculating the difference between the withincondition effect sizes of CT and IPT. Effect sizes were classified as being small $( \pm 0.2)$, medium $( \pm 0.5)$ and large $( \pm 0.8$; Cohen, 1988$)$. In addition, we explored the pattern of change over the course of treatment and the degree to which there was variability in the data by inspecting descriptive statistics of change scores and by calculating Pearson's correlations between scores on each measure at different time points (e.g., IIP baseline with IIP 3 months).

Pearson's correlations were also used to explore the relation between (change in) the candidate mediators and (change in) depression severity. First, we calculated correlations between the BDI-II scores at the different time points ( $0,3,7$ months) and scores on the mediator variables at each of the three time points (crosssectional correlations). We then estimated correlations between change scores on the BDI-II and change indexes of the various mediator measures (longitudinal correlations). In order to do this, we computed change scores on the BDI-II and each of the candidate mediators from baseline to 3 months (pre- to mid-treatment), from 3 to 7 months (mid- to post-treatment) and from baseline to 7 months (entire course of treatment). To facilitate the interpretation of findings, change scores were calculated in such a way that a positive change score always reflected improvement and a negative change score reflected worsening ${ }^{2}$. As a result, a positive correlation between two change scores indicates that improvement in depression severity is related to improvement on the mediator measure (and vice versa). Negative correlations between change scores, on the other hand, indicate that improvement in depression severity is related to deterioration on the mediator measure (and vice versa). In evaluating longitudinal correlations, we made a distinction between temporal and concurrent relations. Temporal relations represent correlations between change in the mediator and change in the BDI-II that precede or follow those changes (e.g., correlation of change in alliance from 0 to 3 months with change in depression from 3 to 7 months). In case of a concurrent relation, the correlation was estimated of a change score on one measure with change on another measure over the same time period (e.g., change in depression from 0 to 3 months associated with change in cognitions from 0 to 3 months). In addition, we examined the correlations between (change) scores on the BDI-II at different timepoints. Correlations were classified as being small $( \pm 0.1)$, medium $( \pm 0.3)$ and large $( \pm 0.5)$.

To examine the various relations between change in the mediator measures and change in depressive symptoms more closely, mediation analyses for longitudinal data were performed. A simple method to assess mediation in longitudinal data is to compute preto post-treatment change scores in mediator measure and outcome, and then use these scores as variables in a single mediation model (MacKinnon, 2008). However, this method has some drawbacks for use in the current study. First of all, it only includes pre- and post-treatment scores, hereby ignoring information collected at 3-months (mid-treatment). As a result, it is not possible to take change from pre-to mid-treatment (baseline- 3 months) and from mid-to post-treatment (3-7 months) into account. Second, this method assumes that change is constant over time, hereby not considering the possibility that treatment effects may vary in different phases of treatment (e.g., more change in the early phase, and less change in the late phase, or vice versa). However, it would also not be ideal to calculate difference scores for each time period separately (baseline-3 months and 3-7 months) and then use these scores in the model, because this might lead to problems of compounding measurement error. Latent Difference Score (LDS) models overcome these drawbacks. LDS models use latent instead of observed difference scores to test questions about temporal relationships between variables, which are explicitly defined as part of the model, and estimate their magnitude. Latent difference scores are defined as that part of the measurement that is not identical to the score obtained at the previous measurement, and are without measurement error. Given our repeated measures design, the fact that the magnitude of change differed in the early and late phase of treatment, and because we wanted to minimize measurement unreliability, we considered LDS models best suited for answering our research questions (see McArdle, 2009).

LDS models are estimated under the Structural Equation Modeling (SEM) framework. For the current study we used the

\footnotetext{
${ }^{2}$ First, scores on the SLSC-R and the WAI-O-S (for which higher scores reflect better outcomes) were reversed coded, so that, for each measure, a lower score was indicative for a better outcome. After that, change scores $(0-3,3-7,0-7)$ were calculated by subtracting scores on time-point $t+1$ from scores on time-point $t$ (e.g., IIP 3 months - IIP 7 months)
} 
LDS model proposed by MacKinnon (2008). This model tests the relation between treatment and outcome ( $c$ path), the relation between treatment and mediator ( $a$ path), and the set of possible concurrent and temporal relations between mediator and outcome ( $b$ paths). Fig. 2 illustrates the proposed model. Y represents the outcome variable BDI-II. $\mathrm{M}$ represents the candidate mediator (DAS-A17 in this example) and X refers to the contrast between CT and IPT. The squares in the model represent the observed raw scores at each time point. Circles represent latent variables (defined as the difference between the scores on two consecutive assessments). The $b_{1}$ and $b_{2}$ paths represent concurrent relationships between mediator and outcome for $0-3$ and 3-7 months respectively. Path $b_{3}$ and $b_{4}$ illustrate temporal relationships. $b_{3}$ refers to the relationship between previous levels of the mediator and subsequent changes in the outcome (e.g., mediator at 3 months and subsequent change in depression from 3 to 7 months). To conclude, $b_{4}$ illustrates the relationship between change in the mediator at time t-1 and change in the outcome at time $t$. In order to obtain the latent difference scores, two paths in the model - the paths from time 1 to time 2 , and the paths from the latent difference to time 2 - were set to 1 (MacKinnon, 2008) ${ }^{3}$.

We ran five LDS models, one for each of the mediator measures. In each model, all relations between the mediator and outcome were tested. A positive relationship indicates that more change in the mediator is associated with more change in the outcome. If the relation is negative, it means that more change in the mediator is associated with less change in the outcome. In order to rule out reversed causality (i.e., symptom change facilitates change in the mediator), we reran our analyses but now added the relation between change in symptoms from ( $\Delta$ BDI-II 0-3 months) and subsequent change in the mediator ( $\Delta$ in mediator $3-7$ ) to the model, and compared its fit with the fit of the model without this relation.

As a last step, the presence and magnitude of mediation was determined by estimating the various indirect (mediating) effects: the product of the coefficients of paths $a^{*} b$. Indirect effects represent the effect of treatment on outcome that goes through the mediator (e.g., $a_{1}{ }^{*} b_{4}$, is the effect of treatment on change in depression in the period 3-7 that is produced by change in DASA17 in the period 0-3; MacKinnon, 2008; Muthén \& Asparouhov, 2014). We used bias corrected $95 \%$ bootstrap confidence intervals (CI) with 1000 bootstrap samples to assess the parameter estimates. If zero was not contained in the confidence intervals we concluded that the indirect effect was significant. Indirect effects referred to effects in both treatments. When an indirect effect was found to be significant, we explored the differences between the two treatment conditions by estimating the various $b$ paths separately for CT and IPT.

\section{Results}

\subsection{Descriptive statistics}

Table 1 presents the mean (SD) scores on the outcome variable BDI-II and on all candidate mediators at each time-point in the treatment phase $(0,3,7$ months). Furthermore, effect sizes over the course of treatment are presented. All data were normally distributed, except for WAI-O-S at 3 months (Kurtosis $=2.14$ ). Reliability coefficients ranged from $\alpha=0.87$ (BDI-II) to $\alpha=0.94$ (WAI-O-S). CT

\footnotetext{
3 This was done in order to mimic the result of a subtraction (e.g., RRS 3 months $=1 *$ RRS baseline $+1 * \Delta$ RRS baseline and 3 months) (McArdle, 2009).

${ }^{4}$ Because further analyses included difference scores, we did not control for baseline differences.
}

and IPT showed significant baseline differences on the RRS ( $t$ $(149)=-2.4, p=0.02)$ and the WAI-O-S $(t(81.2)=3.5, p<0.01)^{4}$. Over the course of treatment, the means of all measures changed in the expected direction. Pre-to post-treatment (0-7 months) effect sizes were large $(d>0.80)$ for BDI-II and RRS, medium to large $(0.5<d<0.8)$ for DAS-A17, SLSC-R and IIP, and small to medium $(0.20<d<0.50)$ for the WAI-O-S. Between-group effect sizes were small (range 0.06-0.19), except for the IIP $(d=0.30)$. Change in DAS-A-17, IIP, RRS, and SLSC mainly occurred in the second half of treatment (3-7 months; see Table 1). Correlations between scores on mediator measures at different time points (e.g., correlation between DAS-A-17 score at baseline and three months) ranged from 0.65 to 0.70 .

\subsection{Relationships between (change in) mediator measures and (change in) depression severity}

\subsubsection{Correlation analyses}

Table 2 presents Pearson's correlations between (change in) depression severity (BDI-II) and (change) scores on the five candidate mediators. Correlations between (change) scores on the BDI-II at different time-points are provided as well. Scores on the BDI-II and the various mediator measures were highly correlated (both cross-sectionally and longitudinally). Crosssectional correlations showed that, at each time-point, higher levels of depression severity were associated with more dysfunctional attitudes (DAS-A17), more interpersonal problems (IIP), higher levels of rumination (RRS); and lower levels of selfesteem (SLSC-R) and alliance (WAI-O-S). It has to be noted that the correlations between alliance and depression were small and (mostly) non-significant. In line with cross-sectional findings, longitudinal correlations indicated that improvement in depression severity was associated with improvement in the mediator measures. The largest longitudinal correlations were found for the concurrent relations over the entire course of treatment $(0-7$, cells with light grey shade), with correlations ranging from 0.33 to 0.60 . A closer look at these correlations indicated that this was mainly the result of high correlations in the second part of treatment (3-7 months). The temporal relations between BDI-II and the mediator measures were found to be small and nonsignificant (cells shaded dark grey). Examination of the correlations between (change) scores on the BDI-II at different timepoints showed that the temporal relation was negative, indicating that a strong improvement in depressive symptoms in the early phase of therapy (0-3) was associated with less improvement later on (3-7), and vice versa.

\subsubsection{Latent difference score models}

Beta coefficients and standard errors of the various relations in the five LDS models are displayed in Table 3. The models for cognitions (DAS-A17) and alliance (WAI-O-S) showed a good fit, whereas the other models showed a poor to reasonable fit (Kline, $2005)^{5}$. Reflecting the fact that no main effect of treatment condition was found (Lemmens et al., 2015), standardized regression coefficients representing relations between treatment and outcome ( $c$ paths) were all non-significant. Examination of the relation between treatment and change in the mediators ( $a$ paths) revealed only a significant difference between CT and IPT on the IIP, indicating that changes on the IIP were different for CT and IPT

\footnotetext{
${ }^{5} \chi 2=5.05(\mathrm{p}=0.17)$, RMSEA $=0.07, \mathrm{CFI}=1.00$ for DAS-A-NL; $\chi 2=11.69$ $(\mathrm{p}=0.01)$, RMSEA $=0.14, \mathrm{CFI}=0.98$ for IIP; $\chi 2=9.43(\mathrm{p}=0.02)$, RMSEA $=0.12$, CFI $=0.98$ for RRS; $\chi 2=18.13(\mathrm{p}=0.00)$, RMSEA $=0.18, \mathrm{CFI}=0.96$ for SLSC-R; $\chi^{2}=3.05(\mathrm{p}=0.38), \mathrm{RMSEA}=0.01, \mathrm{CFI}=1.00$ for WAI-O-S.
} 


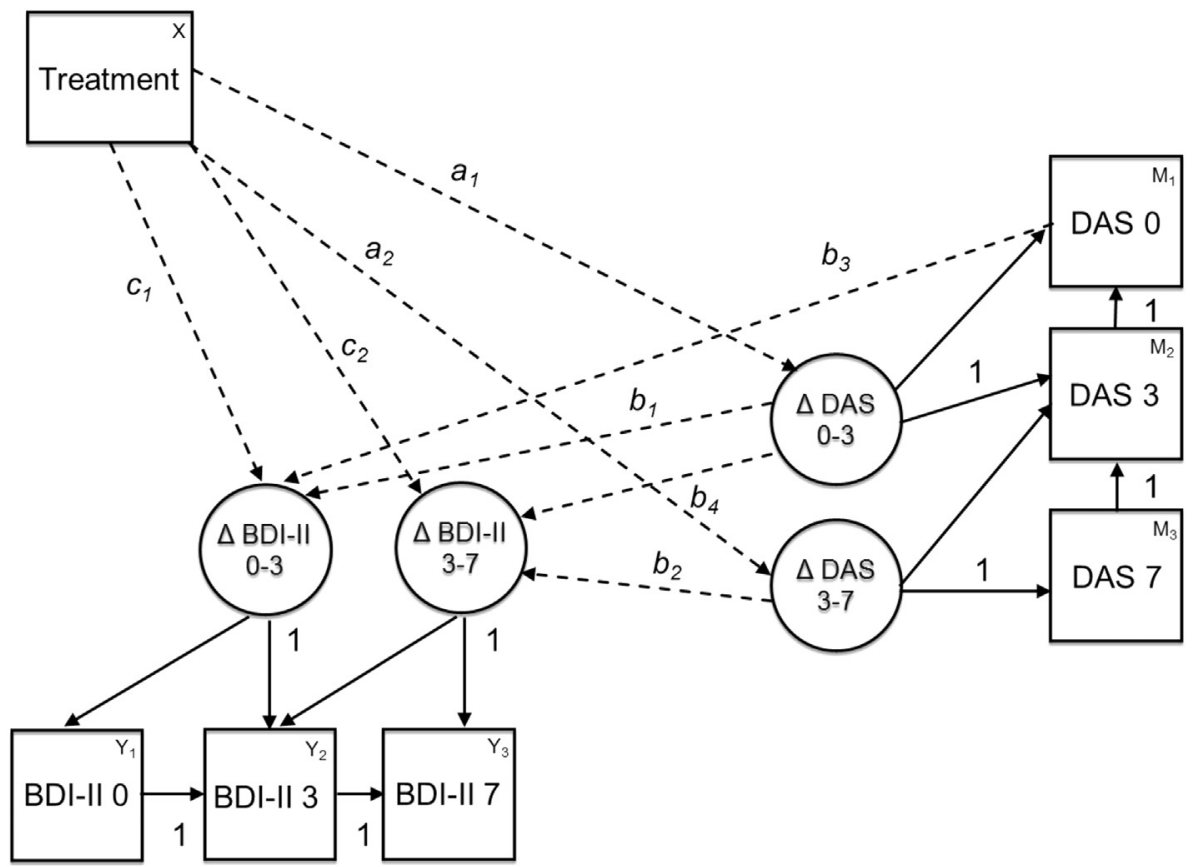

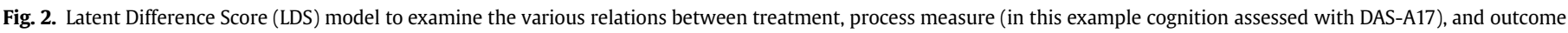

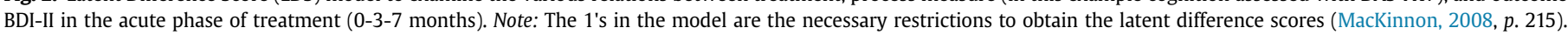

Table 1

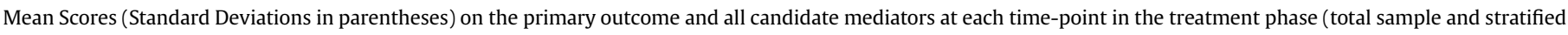
per condition).

\begin{tabular}{|c|c|c|c|c|c|}
\hline & \multirow{2}{*}{$\begin{array}{l}\text { Baseline } \\
\text { Mean (SD) }\end{array}$} & \multirow{2}{*}{$\begin{array}{l}3 \text { months } \\
\text { Mean (SD) }\end{array}$} & \multirow{2}{*}{$\begin{array}{l}7 \text { months } \\
\text { Mean (SD) }\end{array}$} & \multicolumn{2}{|l|}{ Effect size $d$} \\
\hline & & & & Within-Groups ${ }^{\mathrm{a}}$ & CT vs. IPT ${ }^{b}$ \\
\hline \multicolumn{6}{|c|}{ Outcome variable } \\
\hline \multicolumn{6}{|c|}{ Depression Severity (BDI-II) } \\
\hline Total & $29.8(9.0)$ & $23.2(12.0)$ & $14.8(12.1)$ & 1.67 & \\
\hline $\mathrm{CT}$ & $28.4(8.9)$ & $22.4(11.0)$ & $13.7(10.8)$ & $1.65^{c}$ & \\
\hline IPT & $31.2(8.9)$ & $24.1(13.0)$ & $16.0(13.4)$ & 1.71 & -0.06 \\
\hline \multicolumn{6}{|c|}{ Mediator variables } \\
\hline \multicolumn{6}{|c|}{ Dysfunctional Attitudes (DAS-A17) } \\
\hline Total & $62.0(16.0)$ & $60.3(16.8)$ & $53.1(16.0)$ & 0.56 & \\
\hline $\mathrm{CT}$ & $61.1(15.4)$ & $60.0(15.0)$ & $51.4(14.7)$ & 0.63 & \\
\hline IPT & $62.9(16.6)$ & $60.7(18.6)$ & $54.9(17.3)$ & 0.48 & 0.15 \\
\hline \multicolumn{6}{|c|}{ Interpersonal Problems (IIP) } \\
\hline Total & $86.4(29.7)$ & $81.3(32.6)$ & $67.4(36.7)$ & 0.64 & \\
\hline $\mathrm{CT}$ & $83.1(24.7)$ & $82.8(28.1)$ & $62.9(36.3)$ & 0.82 & \\
\hline IPT & 89.7 (33.9) & $79.8(36.9)$ & $72.0(36.8)$ & 0.52 & 0.30 \\
\hline \multicolumn{6}{|c|}{ Rumination (RRS) } \\
\hline Total & $50.7(8.9)^{*}$ & $47.9(10.5)$ & $42.0(11.5)$ & 0.98 & \\
\hline $\mathrm{CT}$ & $49.1(9.1)$ & $47.0(9.7)$ & $40.9(10.9)$ & 0.90 & \\
\hline IPT & $52.4(8.4)$ & $48.9(11.2)$ & $43.2(12.1)$ & 1.10 & -0.19 \\
\hline \multicolumn{6}{|c|}{ Self-esteem (SLSC-R) } \\
\hline Total & $38.4(9.7)$ & $39.7(9.8)$ & $44.2(11.2)$ & 0.60 & \\
\hline $\mathrm{CT}$ & $39.3(8.6)$ & $39.8(9.4)$ & $44.9(10.6)$ & 0.65 & \\
\hline IPT & $37.6(10.7)$ & $39.6(10.4)$ & $43.4(11.9)$ & 0.54 & 0.11 \\
\hline \multicolumn{6}{|c|}{ Alliance (WAI-O-S) } \\
\hline Total & $65.0(7.4)^{*}$ & $66.7(7.8)^{*}$ & $67.9(10.3)^{*}$ & 0.39 & \\
\hline $\mathrm{CT}$ & $67.8(5.2)$ & $68.7(6.3)$ & $70.4(8.6)$ & 0.50 & \\
\hline IPT & $62.7(8.1)$ & $64.6(8.7)$ & $65.2(11.3)$ & 0.31 & 0.19 \\
\hline
\end{tabular}

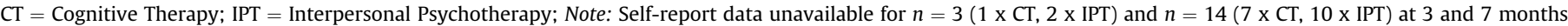

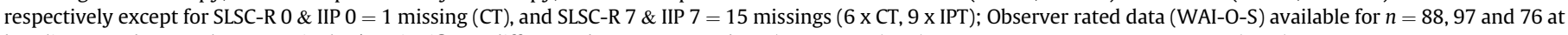

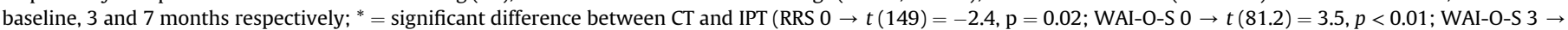
$t(95)=2.6, p=0.01$; WAI-O-S $7 \rightarrow t(74)=2.2, p=0.03)$.

a $\left(\mathrm{M} \mathrm{t}_{0}-\mathrm{M} \mathrm{t}_{7}\right) / \mathrm{SD} \mathrm{t}_{0}$.

b Difference in improvement effect sizes between CT and IPT.

c The careful reader will notice that the effect sizes on the BDI-II that are reported here are slightly different from those reported in our main outcome paper $(d=1.71$ for CT

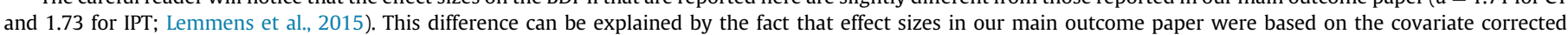
multilevel estimates (baseline scores on BDI/EQ5D), whereas in the current paper observed values were used. 
Table 2

Pearson's correlations between (changes in) depression severity (BDI-II) and (changes in) scores on the five candidate mediator measures (total sample).

\begin{tabular}{|c|c|c|c|c|c|c|c|c|c|c|c|c|c|c|c|}
\hline \multicolumn{8}{|c|}{ Depression (BDI-II) } & \multicolumn{8}{|c|}{ Depression (BDI-II) } \\
\hline & & $0 \mathrm{~m}$ & $3 \mathrm{~m}$ & $7 \mathrm{~m}$ & $\Delta 0 \rightarrow 3$ & $\Delta 3 \rightarrow 7$ & $\Delta 0 \rightarrow 7$ & & & $0 \mathrm{~m}$ & $3 \mathrm{~m}$ & $7 \mathrm{~m}$ & $\Delta 0 \rightarrow 3$ & $\Delta 3 \rightarrow 7$ & $\Delta 0 \rightarrow 7$ \\
\hline \multirow{6}{*}{$\begin{array}{l}\text { Depression } \\
\text { (BDI-II) }\end{array}$} & $0 \mathrm{~m}$ & 1 & $0.63^{* *}$ & $0.45^{* *}$ & 0.15 & $0.20^{*}$ & $0.30^{* *}$ & \multirow{6}{*}{$\begin{array}{l}\text { Rumination } \\
\text { (RRS) }\end{array}$} & $0 \mathrm{~m}$ & $0.50^{* *}$ & $0.40^{* *}$ & $0.20^{*}$ & -0.03 & $0.20^{*}$ & 0.16 \\
\hline & $3 \mathrm{~m}$ & - & 1 & $0.66^{* *}$ & $-0.67^{* *}$ & $0.40^{* *}$ & $-0.22^{*}$ & & $3 \mathrm{~m}$ & $0.50^{* *}$ & $0.60^{* *}$ & $0.35^{* *}$ & $-0.29^{* *}$ & $0.25^{* *}$ & 0.00 \\
\hline & $7 \mathrm{~m}$ & - & - & 1 & $-0.40^{* *}$ & $-0.43^{* *}$ & $-0.72^{* *}$ & & $7 \mathrm{~m}$ & $0.26^{* *}$ & $0.46^{* *}$ & $0.65^{* *}$ & $-0.34^{* *}$ & $-0.23^{* *}$ & $-0.49^{* *}$ \\
\hline & $\Delta 0 \rightarrow 3$ & - & - & - & 1 & $-0.32^{* *}$ & $0.55^{* *}$ & & $\Delta 0 \rightarrow 3$ & -0.10 & $-0.34^{* *}$ & $-0.20^{*}$ & $0.34^{* *}$ & -0.09 & $0.18^{*}$ \\
\hline & $\Delta 3 \rightarrow 7$ & - & - & - & - & 1 & $0.61^{* *}$ & & $\Delta 3 \rightarrow 7$ & 0.17 & 0.03 & $-0.40^{* *}$ & 0.12 & $0.52^{* *}$ & $0.56^{* *}$ \\
\hline & $\Delta 0 \rightarrow 7$ & - & - & - & - & - & 1 & & $\Delta 0 \rightarrow 7$ & 0.12 & -0.16 & $-0.47^{* *}$ & $0.31^{* *}$ & $0.38^{* *}$ & $0.60^{* *}$ \\
\hline \multirow{6}{*}{$\begin{array}{l}\text { Dysf. Attitudes } \\
\text { (DAS-A17) }\end{array}$} & $0 \mathrm{~m}$ & $0.43^{* *}$ & $0.32^{* *}$ & $0.21^{*}$ & 0.00 & 0.16 & 0.15 & \multirow{6}{*}{$\begin{array}{l}\text { Self-Esteem } \\
\text { (SLSC-R) }\end{array}$} & $0 \mathrm{~m}$ & $-0.41^{* *}$ & $-0.34^{* *}$ & -0.16 & 0.05 & $-0.22^{*}$ & -0.17 \\
\hline & $3 \mathrm{~m}$ & $0.22^{* *}$ & $0.18^{*}$ & 0.16 & -0.02 & 0.06 & 0.04 & & $3 \mathrm{~m}$ & $-0.52^{* *}$ & $-0.55^{* *}$ & $-0.26^{* *}$ & $0.20^{*}$ & $-0.34^{* *}$ & -0.14 \\
\hline & $7 \mathrm{~m}$ & $0.26^{* *}$ & $0.29^{* *}$ & $0.52^{* *}$ & -0.12 & $-0.29^{* *}$ & $-0.35^{* *}$ & & $7 \mathrm{~m}$ & $-0.42^{* *}$ & $-0.48^{* *}$ & $-0.53^{* *}$ & $0.22^{*}$ & 0.05 & $0.23^{* *}$ \\
\hline & $\Delta 0 \rightarrow 3$ & $0.26^{* *}$ & $0.17^{* *}$ & 0.05 & 0.03 & 0.12 & 0.14 & & $\Delta 0 \rightarrow 3$ & -0.14 & $-0.24^{* *}$ & -0.12 & $0.18^{*}$ & -0.15 & 0.02 \\
\hline & $\Delta 3 \rightarrow 7$ & 0.03 & -0.07 & $-0.41^{* *}$ & 0.11 & $0.42^{* *}$ & $0.46^{* *}$ & & $\Delta 3 \rightarrow 7$ & 0.08 & 0.02 & $-0.39^{* *}$ & 0.06 & $0.47^{* *}$ & $0.47^{* *}$ \\
\hline & $\Delta 0 \rightarrow 7$ & $0.23^{* *}$ & 0.07 & $-0.33^{*}$ & 0.13 & $0.48^{* *}$ & $0.53^{* *}$ & & $\Delta 0 \rightarrow 7$ & -0.05 & $-0.21^{*}$ & $-0.46^{* *}$ & $0.22^{*}$ & $0.30^{* *}$ & $0.45^{* *}$ \\
\hline \multirow{6}{*}{$\begin{array}{l}\text { Int. Problems } \\
\text { (IIP) }\end{array}$} & $0 \mathrm{~m}$ & $0.48^{* *}$ & $0.40^{* *}$ & $0.30^{* *}$ & -0.04 & 0.10 & 0.06 & \multirow{6}{*}{$\begin{array}{l}\text { Alliance } \\
\text { (WAI-O-S) }\end{array}$} & $0 \mathrm{~m}$ & -0.14 & -0.04 & -0.17 & -0.07 & 0.16 & 0.08 \\
\hline & $3 \mathrm{~m}$ & $0.44^{* *}$ & $0.45^{* *}$ & $0.31^{* *}$ & -0.16 & $0.19^{*}$ & 0.01 & & $3 \mathrm{~m}$ & -0.10 & -0.16 & $-0.30^{* *}$ & 0.12 & 0.16 & 0.28 \\
\hline & $7 \mathrm{~m}$ & $0.34^{* *}$ & $0.47^{* * *}$ & $0.62^{* *}$ & $-0.28^{* *}$ & $-0.18^{*}$ & $-0.39^{* *}$ & & $7 \mathrm{~m}$ & -0.22 & $-0.29^{*}$ & $-0.37^{* *}$ & 0.15 & 0.13 & $0.23^{*}$ \\
\hline & $\Delta 0 \rightarrow 3$ & 0.01 & -0.12 & -0.05 & $0.17^{*}$ & -0.14 & 0.06 & & $\Delta 0 \rightarrow 3$ & 0.09 & -0.08 & -0.14 & 0.17 & 0.04 & $0.24^{*}$ \\
\hline & $\Delta 3 \rightarrow 7$ & 0.05 & -0.08 & $-0.44^{* *}$ & 0.14 & $0.44^{* *}$ & $0.51^{* *}$ & & $\Delta 3 \rightarrow 7$ & $-0.29^{*}$ & -0.21 & -0.23 & -0.01 & 0.05 & 0.03 \\
\hline & $\Delta 0 \rightarrow 7$ & 0.06 & $-0.20^{*}$ & $-0.46^{* *}$ & $0.31^{* *}$ & $0.31^{* *}$ & $0.53^{* *}$ & & $\Delta 0 \rightarrow 7$ & -0.03 & $-0.26^{*}$ & $-0.32^{*}$ & $0.31^{*}$ & 0.10 & $0.33^{* *}$ \\
\hline
\end{tabular}

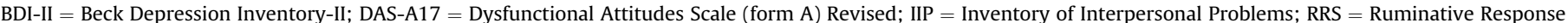

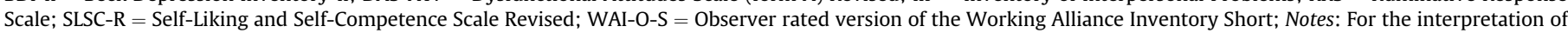

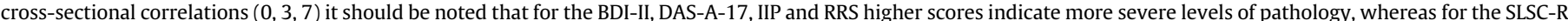

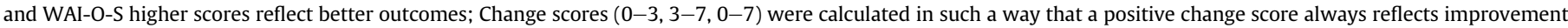

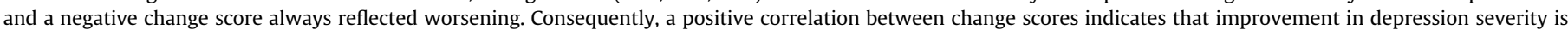

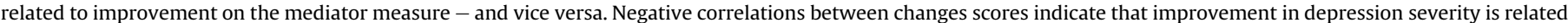

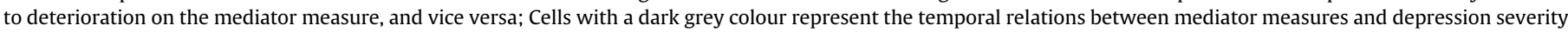
(BDI-II). Cells with a light grey shade represent concurrent relations between mediator measures and depression severity (BDI-II); ${ }^{*} p<0.05 ;{ }^{* *} p<0.01$.

in different phases of treatment. IPT showed a larger decrease in Interpersonal problems in the first half of treatment (0-3 months), whereas CT showed a larger change in the second half (3-7 months: see Table 1 for means and SD's). Over the entire course of treatment (0-7 months), these effects cancelled each other out (total change on the IIP of 20.2 points in CT vs. 17.7 in IPT).

Various relations were found between change in candidate mediators and change in depression ( $b$ paths). First of all, in both phases of therapy (0-3 and 3-7 months), significant concurrent relations ( $b_{1}$ and $b_{2}$ paths) were found between change in depression severity and changes in Interpersonal problems (IIP), Rumination (RRS) and Self-esteem (SLSC-R). For the DAS-A17, only a concurrent relation in the second phase of treatment was found $\left(b_{2}\right)$. All beta coefficients were positive, indicating that more change in the mediator variable was associated with more change in the outcome. The WAI-O-S did not show any significant concurrent relations. Second, $b_{3}$ paths in the second half of treatment were significant for the DAS-A17, the IIP, the RRS and the WAI-O-S. This demonstrates that levels at mid-treatment (3 months) of dysfunctional attitudes, interpersonal problems, rumination, and alliance, were related to change in depression severity in the period thereafter (3-7 months) ${ }^{6}$. Again, all beta coefficients were positive. Third and finally, a significant positive temporal relation between early change in the SLSC-R (0-3) and subsequent change on the BDI-II ( $3-7 ; b_{4}$ path) was found, indicating that change in selfesteem preceded change in depression severity. Adding the reversed temporal relations to the models resulted in only minimal change in model fit estimates, indicating that the complex models were not better than the fit of the simple models. It was therefore concluded that the reversed paths did not show substantial temporal relations.

\footnotetext{
${ }^{6}$ The $b_{3}$ paths in the early phase of treatment (mediator at 0 to change in depression from 0 to 3 ), were all non-significant, and therefore set to zero.
}

\subsection{Indirect effects}

As shown in Table 3, we found three significant indirect (mediating) effects $\left(a^{*} b\right)$, all on the IIP. The significant $a^{*} b_{3}$ path (95\% CI from -1.60 to -0.05 ) indicates that change on the BDI-II in the early phase of treatment ( $0-3)$, was mediated by initial levels of IIP. Furthermore, change on the BDI-II was mediated by concurrent change on the IIP both in the early phase $\left(a^{*} b_{1}: 95 \% \mathrm{CI}\right.$ from -2.01 to -0.06 ) and late phase of treatment (3-7 months $\mathrm{a}^{*} \mathrm{~b}_{2}$ : $95 \% \mathrm{CI}$ from 0.19 to 4.06$)$. No temporal mediation $\left(a^{*} b_{4}\right)$ was found $(95 \% \mathrm{CI}$ of -0.97 to 0.52 ). Exploration of the various $b$ paths separately for CT and IPT (Table 4) suggests that the relation between changes in interpersonal functioning and depression severity was different for these treatments. For IPT there was a concurrent relationship between change in IIP and changes in BDI from 3 to 7 months, and evidence for a negative longitudinal relationship ( $b_{4}$ path). For $C T$ we only found concurrent relationships. Furthermore, in CT the (non-significant) longitudinal relationship ( $b_{4}$ path) was positive. No significant indirect effects were found on the other measures.

\section{Discussion}

The current study examined change in five hypothesized working mechanisms of CT and IPT - two presumably different treatments for MDD - in relation to change in depressive symptoms over the course of treatment. It also examined whether the pattern in the data was consistent with theorized causal mediation models. The unique (temporal) features of our study design and the use of LDS models allowed us to expand on previous (mostly crosssectional) research on mechanisms.

In line with our expectations, patients' scores on all investigated measures changed for the better over the course of treatment, with medium to large effect sizes. Largest changes were found in depressive symptoms and rumination. The quality of working alliance showed the least change. It should be noted that alliance scores were already very high to begin with - hereby leaving only 
Table 3

Unstandardized coefficients (standard errors in parentheses) of the five Latent Difference Score (LDS) Models.

\begin{tabular}{|c|c|c|c|c|c|c|}
\hline & & $\begin{array}{l}\text { Dysfunctional Attitudes } \\
\text { (DAS-A17) B (SE) }\end{array}$ & $\begin{array}{l}\text { Interpersonal Problems (IIP) } \\
\text { B (SE) }\end{array}$ & $\begin{array}{l}\text { Rumination (RRS) } \\
\text { B (SE) }\end{array}$ & $\begin{array}{l}\text { Self-Esteem (SLSC-R) } \\
\text { B (SE) }\end{array}$ & $\begin{array}{l}\text { Alliance (WAI-O-S) } \\
\text { B (SE) }\end{array}$ \\
\hline Direct & $\mathrm{c}_{1}$ & $-0.40(1.45)$ & $0.29(1.52)$ & $-0.21(1.35)$ & $0.16(1.35)$ & $-0.41(1.81)$ \\
\hline \multirow[t]{7}{*}{ Effects } & $\mathrm{c}_{2}$ & $0.93(1.24)$ & $-0.31(1.35)$ & $0.85(1.27)$ & $1.02(1.35)$ & $-0.11(1.69)$ \\
\hline & $a_{1}$ & $-0.31(1.87)$ & $-9.28(3.74)^{*}$ & $-1.00(1.29)$ & $-1.54(1.19)$ & $0.88(1.20)$ \\
\hline & $a_{2}$ & $1.72(2.04)$ & $9.79(4.48)^{*}$ & $1.59(1.55)$ & $1.10(1.41)$ & $0.63(1.61)$ \\
\hline & $\mathrm{b}_{1}$ & $0.00(0.07)$ & $0.09(0.03)^{* *}$ & $0.52(0.12)^{* * *}$ & $0.42(0.13)^{* *}$ & $0.28(0.16)^{+}$ \\
\hline & $b_{2}$ & $0.46(0.05)^{* * *}$ & $0.20(0.03)^{* * *}$ & $0.57(0.08)^{* * *}$ & $0.62(0.08)^{* * *}$ & $0.17(0.15)$ \\
\hline & $b_{3}$ & $0.16(0.05)^{* *}$ & $0.07(0.03)^{*}$ & $0.20(0.10)^{*}$ & $-0.04(0.08)$ & $0.38(0.16)^{*}$ \\
\hline & $\mathrm{b}_{4}$ & $0.11(0.06)^{+}$ & $0.02(0.03)$ & $0.05(0.09)$ & $0.22(0.10)^{*}$ & $-0.09(0.21)$ \\
\hline Indirect & $a^{*} b_{1}$ & $0.00(0.14)$ & $-0.84(0.45)^{\mathrm{a}}$ & $-0.52(0.68)$ & $-0.64(0.61)$ & $0.25(0.40)$ \\
\hline \multirow[t]{3}{*}{ Effects } & $a^{*} b_{2}$ & $0.79(0.98)$ & $1.94(0.92)^{\mathrm{a}}$ & $0.91(0.93)$ & $0.68(0.90)$ & $0.11(0.37)$ \\
\hline & $a^{*} b_{3}$ & $-0.05(0.31)$ & $-0.63(0.35)^{\mathrm{a}}$ & $-0.20(0.31)$ & $0.06(0.19)$ & $0.34(0.51)$ \\
\hline & $a^{*} b_{4}$ & $-0.04(0.26)$ & $-0.15(0.31)$ & $-0.05(0.15)$ & $-0.33(0.29)$ & $-0.08(0.33)$ \\
\hline
\end{tabular}

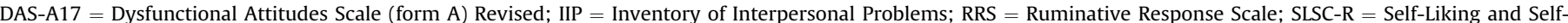

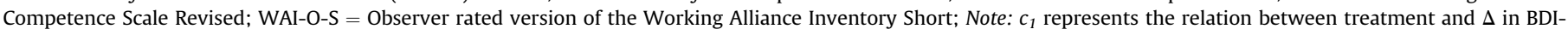

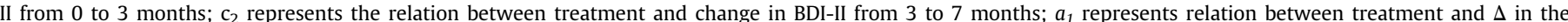

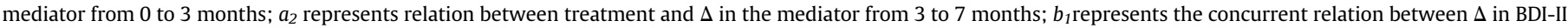

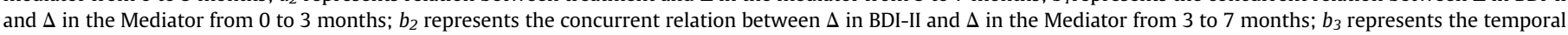

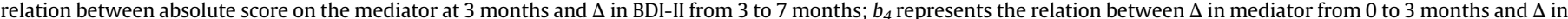

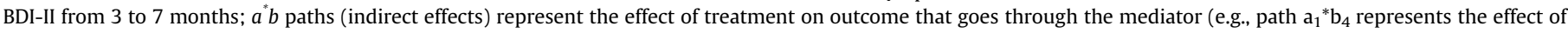

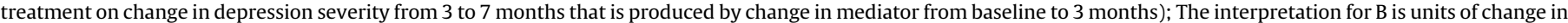

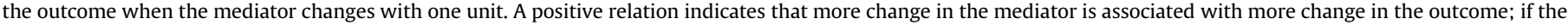
relationship is negative, it means that more change in the mediator is associated with less change in the outcome; $-+-p<0.10,{ }^{*} p<0.05 ;{ }^{* *} p<0.01 ;{ }^{* * *} p<0.001$.

a Significant indirect effect based on $95 \% \mathrm{CI}$ with 1000 bootstrap samples ( -2.01 to -0.06 for $a^{*} b_{1} ; 0.19$ to 4.06 for $a^{*} b_{2}$; and -1.60 to -0.05 for $a^{*} b_{3}$ ).

Table 4

Unstandardized coefficients (standard errors) of the various relations between changes in Interpersonal Problems (IIP) and Depressive Symptoms (BDI-II), separately for CT and IPT.

\begin{tabular}{llll}
\hline & & $\begin{array}{l}\text { Cognitive Therapy } \\
\mathrm{B}(\mathrm{SE})\end{array}$ & $\begin{array}{l}\text { Interpersonal Psychotherapy } \\
\mathrm{B}(\mathrm{SE})\end{array}$ \\
\hline \multirow{2}{*}{ Effects } & $\mathrm{b}_{1}$ & $0.09(0.04)^{*}$ & $0.02(0.06)$ \\
& $\mathrm{b}_{2}$ & $0.20(0.03)^{* * *}$ & $0.21(0.06)^{* * *}$ \\
& $\mathrm{~b}_{3}$ & $0.02(0.03)$ & $0.07(0.04)$ \\
& $\mathrm{b}_{4}$ & $0.02(0.04)$ & $-0.02(0.06)^{*}$ \\
\hline
\end{tabular}

$\mathrm{CT}=$ Cognitive Therapy; IPT = Interpersonal Psychotherapy; BDI-II = Beck Depression Inventory-II; IIP $=$ Inventory for Interpersonal Problems; $b_{1}$ represents the concurrent relation between $\Delta$ in BDI-II and $\Delta$ in IIP from 0 to 3 months (early phase); $b_{2}$ represents the concurrent relation between $\Delta$ in BDI-II and $\Delta$ in IIP from 3 to 7 months (late phase); $b_{3}$ represents the temporal relation between absolute score on the IIP at 3 months and $\Delta$ in BDI-II from 3 to 7 months (late phase); $b_{4}$ represents the relation between $\Delta$ in IIP from 0 to 3 months and $\Delta$ in BDI-II from 3 to 7 months (temporal relation); The interpretation for $B$ is units of change in the outcome when the mediator changes with one unit. A positive relation indicates that more change in the mediator is associated with more change in outcome; a negative relationship means that more change in the mediator is associated with less change in outcome; $-+-p<0.10,{ }^{*} p<0.05$; $^{* *} p<0.01$; ${ }^{* * *} p<0.001$.

little room for further improvement - and remained stable over the course of therapy. No differential effects in pre-to post-treatment changes were observed between the two conditions. However, change in interpersonal functioning occurred more rapidly in IPT than in CT, although the latter caught up.

In each of the treatments, concurrent changes in mediators measures and depressive symptoms were strongly related. More specifically, improvement in depression severity was associated with significant concurrent improvement in self-esteem, and with a decrease of dysfunctional attitudes, interpersonal problems, and rumination. Except for a significant relation between early change in self-esteem (0-3) and subsequent change in depression (3-7), no significant temporal relations were found. With regard to indirect (mediation) effects, we found that change on the BDI-II was mediated by concurrent change on the IIP in both phases of treatment. However, we did not find evidence for temporal mediation, hereby limiting the value of our findings. In addition, the model had poor fit.

Exploration of the various $b$ paths separately for CT and IPT indicated that the relation between interpersonal functioning and depression was different for CT and IPT. The negative longitudinal relationship between IIP and depression in IPT might point towards a ceiling (or floor) effect. No evidence for mediation was found on the other mediators.

\subsection{No differences in pre- to post-treatment change on mediators between CT and IPT}

From a theoretical point of view, the fact that patients treated with CT and IPT exhibited a similar degree of improvement on all mediator measures - including cognitive beliefs and interpersonal functioning - might be of interest. According to theory, CT and IPT exert their beneficial effects through their own specific theorized mechanisms. Since IPT, in contrast to CT, does not actively target the modification of dysfunctional cognitions, and CT does not explicitly focus on improvement of interpersonal functioning, one would expect between-condition differences on the therapy-specific mediator measures. However, when taking a closer look at our findings conceptually, there are fair reasons to believe that CT might lead to changes in interpersonal functioning, and that IPT might facilitate cognitive change, both through direct and indirect pathways. For example, even though in IPT beliefs are not systematically examined and challenged, cognitive distortions within an interpersonal context are addressed. Furthermore, because IPT gives the patient the sick role - a temporary status recognizing that depression is a medical illness that keeps the patient from functioning at full capacity - self-blaming cognitions, and feelings of hopelessness and worthlessness are reframed as part of the disorder, rather than being reality-based (see e.g., Bernecker et al., 2014 for more information). Similarly, cognitive interventions and behavioural experiments in CT might have a specific focus on interpersonal situations, thereby directly facilitating change in interpersonal functioning. Indirectly, specific experiences following changes in interpersonal functioning in IPT, might lead to readjustment of schema's and attitudes about the self, world and future. Likewise, decrease of dysfunctional attitudes in CT might also lead to change in beliefs related to interpersonal relationships, making patients more likely to improve their interpersonal 
functioning as well. This line of reasoning is supported by empirical work in this field, which has shown that patients' scores on theorized mediators improve over the course of treatment, but that this is not necessarily specific for one type of treatment (e.g., Backenstrass et al., 2006; Bernecker et al., 2014; Quilty et al., 2008; Renner et al., 2012; Warmerdam et al., 2010).

\subsection{Potential explanations for the limited evidence for the theoretical models of change}

There are several potential explanations for why we only found limited evidence for the theoretical models of change. First of all, it may have been the case that the changes that were observed over the course of therapy were due to spontaneous remission rather than due to treatment. Since we did not include a wait-list control condition over the entire course of therapy, we cannot discard this possibility. However, a comparison between active treatment and wait-list control after two months indicated a relation between treatment and outcome, making this explanation rather unlikely. In line with this, we cannot rule out the possibility that effects were caused by extra-therapeutic changes in the person's life. A second explanation for our findings could be that treatments were not adequately implemented in the sense of mobilizing mechanisms, or were not distinct enough. This explanation seems rather improbable as well since an extensive integrity check confirmed therapy quality and clearly differentiated CT and IPT (see Lemmens et al., 2015). Furthermore, although not picked up in the current study, earlier published studies from this sample do suggest therapyspecific effects. One study indicated that sudden gains - large symptom improvements within a single between-session interval that are assumed to be driven by cognitive change - occurred more frequently in CT than in IPT (Lemmens, DeRubeis, Arntz, Peeters, \& Huibers, 2016). In another study (Huibers et al., 2015), we identified a set of moderators of outcome and showed that profiles of patients responding well to CT differ from those responding well to IPT. As pointed out by Kazdin (2007), the presence of moderators points towards the presence of mediators.

Third, in order for change in a mediator to account for (subsequent) change in depression severity, there needs to be substantial change over the period of observation. The fact that effect sizes for (individual) mediators were smaller than for the BDI-II already indicates that current mediators could only explain a limited amount of the treatment effect. Furthermore, since the average change in mediator measures in the early phase of treatment was rather small, there was only little change from which to predict. This might indicate that - even though we had a fairly large sample size - the treatment provided in our study was not powerful enough. Quilty et al. (2008) - who did claim a mediating role for dysfunctional attitudes - found greater change in depression than we did in our trial (overall differentials of 4.0 BDI-II points in CT and 4.5 in IPT). This suggests Quilty et al. might have provided more powerful versions of CT and IPT than we did, thereby triggering more specific change from one or both modalities. In addition, the modest between-condition differences in change on the mediator measures, may have limited the possibilities for establishing statistical mediation. Fourth, our results could indicate that theories are incorrect, and that other mechanisms - not assessed in this study - are responsible for therapeutic change. For example, the present study did not assess change in activity level, a powerful theoretical candidate mechanism of CT that is believed to play an important role in IPT as well.

Fifth - and in our view most likely - it may be the case that we were unable to identify potential mediators due to limitations in our design and the (selection of) measurement instruments. For example, we may have conceptualized our processes wrong or might have used measures that were not sufficiently sensitive (Johansson et al., 2010). Especially the suitability of the IIP as the potential theory-specific mediator measure for IPT can be questioned. Furthermore, it is possible that if we would have compared each of the treatments to a control group that showed larger differences (e.g., to a waiting-list control arm covering the full treatment period or a non-psychological intervention), we may have had more options to demonstrate a mediating role of the investigated candidate mediators. In addition, results could also be affected by the fact that the outcome measure BDI-II overlapped in item content with the various mediator measures ${ }^{7}$.

Probably the biggest methodological problem in the current study was the timing of assessments. More specifically, we may have waited too long and spaced them too far apart. Therapeutic change often happens rapidly, rather that gradually over the course of treatment ('aha experience'; see review of Aderka, Nickerson, Bøe, \& Hofmann, 2012 for more details). The sequential change in mediator and outcome may therefore take place on a much shorter time-scale than the one that was used in our study. Therefore, it is likely that by the time our assessments took place, change in both mediator measures and outcome had already occurred, erroneously leading to the conclusion that temporal relations between processes and outcomes do not exist. A more fine-grained analysis of change over time would have been helpful here. However, as we discussed in a previous paper (Lemmens, DeRubeis et al., 2016), timing of observations is a difficult and delicate matter. Not the least because it is important to assess potential mediators before the bulk of change in outcome has occurred. If this is not the case, there is only little additional change in outcome to test for mediation. Since clear information about the speed and shape of change is often lacking, the optimal timing is difficult to determine.

\subsection{Methodological considerations and recommendations}

The current study was novel in that it was the first study that allowed for a direct comparison of mediational effects of various candidate mediators between CT and IPT in a large sample of adult depressed outpatients. Furthermore, it is one of the few studies in the field that included repeated assessments of candidate mediators and outcome over the course of therapy. This temporal RCT design and the use of LDS models provided a unique opportunity to explore temporal relations between candidate mediators and outcome. In addition, whereas most studies only examine mediation, we examined temporal mediation by evaluating both temporal relations and mediation in a single model. This is in line with recommendations by e.g., Kazdin (2007, 2009). To conclude, our study is one of the few trials in the field that also examined alternative causal accounts. So even though we were not able to demonstrate temporal mediation, our method could serve as an example for future studies.

As in all research, the current study also has a number of limitations. First, our study was powered to detect differences on our primary outcome BDI-II (Lemmens et al., 2011; 2015), and not on secondary outcomes or process measures. This may have limited the power of the current analyses. Second, several LDS models had poor fit, and only limited data were available for the assessment of therapeutic alliance. Results should therefore be interpreted with caution. Third, because we only examined single mediation models, the order of significance and potential collaboration between theorized mediators (indirect pathways) remains unknown. Fourth and finally, analyses were performed on group-level. It might be the

\footnotetext{
${ }^{7}$ The BDI-II includes items on e.g., negative cognitions (DAS-A17), rumination (RRS) and self-esteem (SLSC-R).
} 
case that (mediating) effects differ for certain (subgroups of) depressed patients.

Given all of the above, the most important requirement for future research is to improve the timing of assessments. Furthermore, future research should continue to test theories of change, since this can narrow down the number of potential mechanisms and provide guidance for future research questions and improvement of study designs. In doing this, studies should include both theorized mediators as well as mediators that are not directly consistent with theory. Fine-grained analysis of change over time in large samples facilitates the examination of both direct and indirect pathways of change, hereby shedding light on the collaboration between candidate mediators. In addition, it is important that researchers keep investing in the evaluation and development of mediator measures. In particular, fundamental research on the validity of these measures should progress. Moreover, research should also focus on within-person variance or homogeneous subgroups of patients, rather than on a single average for the entire sample. To conclude, studies are needed that focus more specifically on the differential effects between CT and IPT in order to better understand the extent to which the processes are therapy specific.

\subsection{In conclusion}

The present study contributes to the developing research field on mechanisms of change of psychotherapy by examining temporal relations between various candidate (specific and non-specific) mediators and depression severity in a randomized comparison of CT and IPT for depression. In spite of a temporal research design and the use of an innovative statistical approach, we found little empirical evidence for the theoretical models of change, most probably because of the timing of assessments. Timing of assessment is therefore an essential requirement for future research.

\section{Funding}

This research institute of Experimental Psychopathology (EPP), the Netherlands, and the Academic Community Mental Health Centre (RIAGG) in Maastricht, the Netherlands funded this research.

\section{Author contributions}

$\mathrm{MH}, \mathrm{AA}, \mathrm{FP}$ and SH designed the trial. MH obtained funding for the study. LL conducted the trial and carried out recruitment and data-collection. $\mathrm{MH}, \mathrm{AA}, \mathrm{FP}, \mathrm{SH}$, and RD supervised throughout the study. LL, FGG and MH performed the data analysis and interpretation. LL drafted the manuscript in close collaboration with FGG. All authors provided critical revisions and have approved the final version of the manuscript.

\section{Conflict of interest}

The authors declare that they have no competing interests.

\section{Acknowledgements}

We wish to acknowledge the contribution of participating patients and therapists at RIAGG Maastricht. Furthermore, we gratefully thank Annie Raven and Annie Hendriks for their assistance during the study.

\section{References}

Aderka, I. M., Nickerson, A., Bøe, H. J., \& Hofmann, S. G. (2012). Sudden gains during psychological treatments of anxiety and depression: a meta-analysis. Journal of Consulting and Clinical Psychology, 80, 93-101.

Andrusyna, T. P., Luborsky, L., Pham, T., \& Tang, T. Z. (2006). The mechanisms of sudden gains in supportive-expressive therapy for depression. Psychotherapy Research, 16(5), 526-535.

Backenstrass, M., Schwarz, T., Fiedler, P., Joest, K., Reck, C., Mundt, C., et al. (2006). Negative mood regulation expectancies, self-efficacy beliefs, and locus of control orientation: Moderators or mediators of change in the treatment of depression? Psychotherapy Research, 16(2), 250-258.

Barber, J. P. (2009). Toward a working through of some core conflicts in psychotherapy research. Psychotherapy Research, 19(1), 1-12.

Baron, R. M., \& Kenny, D. A. (1986). The moderator - mediator variable distinction in social psychological research: Conceptual, strategic, and statistical considerations. Journal of Personality and Social Psychology, 51(6), 1173-1182.

Beck, A. T. (1964). Thinking and depression: Theory and therapy. Archives of General Psychiatry, 10, 561-571.

Beck, A. T. (1987). Cognitive models of depression. Journal of Cognitive Psychotherapy, An International Quarterly, 1, 5-37.

Beck, A. T., Rush, A. J., Shaw, B. F., \& Emery, G. (1979). Cognitive therapy of depression. New York, NY: Guilford Press.

Beck, A. T., Steer, R., \& Brown, G. K. (1996). Beck depression inventory ii: Manual. Boston, MA: Hartcourt Brace.

Bernecker, S. L. (2012). How and for whom does interpersonal psychotherapy for depression work? Psychotherapy Bulletin, 47(2), 13-17.

Bernecker, S. L., Constantino, M. J., Pazzaglia, A. M., Ravitz, P., \& McBride, C. (2014). Patient interpersonal and cognitive changes and their relation to outcome in interpersonal psychotherapy for depression. Journal of Clinical Psychology, 70(6), 518-527.

Bordin, E. S. (1979). The generalizability of the psychanalytic concept of the working alliance. Psychotherapy: Theory, Research, Practice, Training, 16, 252-260.

Castonguay, L. G., Constantino, M. J., \& Holtforth, M. G. (2006). The working alliance: Where are we and where should we go? Psychotherapy: Theory, Research, Practice, Training, 43(3), 271-279.

Clark, D. A., Beck, A. T., \& Alford, B. A. (1999). Scientific foundations of cognitive therapy and therapy of depression. New York: John Wiley \& Sons.

Cohen, J. (1988). Statistical power analysis for the behavioral sciences. Hillsdale, NJ: Erlbaum.

Constantino, M. J., Coyne, A. E., Luukko, E. K., Newkirk, K., Bernecker, S. L., Ravitz, P., et al. (2017). Therapeutic alliance, subsequent change, and moderators of the alliance-outcome association in interpersonal psychotherapy for depression. Advance online publication: Psychotherapy. http://dx.doi.org/10.1037/ pst0000101.

Cuijpers, P., Geraedts, A. S., van Oppen, P., Andersson, G., Markowitz, J. C., \& van Straten, A. (2011). Interpersonal psychotherapy for depression: A meta-analysis. American Journal of Psychiatry, 168, 581-592.

Cuijpers, P., van Straten, A., Andersson, G., \& van Oppen, P. (2008). Psychotherapy for depression in adults: A meta-analysis of comparative outcome studies. Journal of Consulting and Clinical Psychology, 76(6), 909-922.

DeRubeis, R. J., Evans, M. D., Hollon, S. D., Garvey, M. J., Grove, W. M., \& Tuason, V. B. (1990). How does cognitive therapy work? cognitive change and symptom change in cogntive therapy and pharmacotherapy for depression. Journal of Consulting and Clinical Psychology, 58(6), 862-869.

DeRubeis, R., \& Feeley, M. (1990). Determinants of change in cognitive therapy for depression. Cognitive Therapy and Research, 14(5), 469-482.

van der Does, W. (2002). BDI-ii-nl: Handleiding; de nederlandse versie van de beck depressie inventory-ii. Enschede: Ipskamp.

Feeley, M., DeRubeis, R. J., \& Gelfand, L. A. (1999). The temporal relation of adherence and alliance to symptom change in cognitive therapy for depression. Journal of Consulting and Clinical Psychology, 67(4), 578-582.

First, M. B., Spitzer, R. L., Gibbon, M., \& Williams, J. B. W. (1997). Structured clinical interview for DSM-IV axis I disorders (SCID-I). New York, NY: Biometrics Research Department New York State Psychiatric Institute.

Garrat, G., Ingram, R. E., Rand, K. L., \& Sawalani, G. (2007). Cognitive processes in cognitive therapy: Evaluation of the mechanisms of change in the treatment of depression. Clinical Psychology, 14, 224-239.

Gaston, L., Marmar, C. R., Gallagher, D., \& Thompson, L. W. (1991). Alliance prediction of outcome beyond in-treatment symptomatic change as psychotherapy processes. Psychotherapy Research, 1, 104-113.

de Graaf, L. E., Roelofs, J., \& Huibers, M. J. H. (2009). Measuring dysfunctional attitudes in the general population: The DAS-a revised. Cognitive Therapy and Research, 33, 345-355.

Hayes, A. F. (2009). Beyond baron and kenny: Statistical mediation analysis in the new millennium. Communication Monographs, 76, 408-420.

Hollon, S. D., DeRubeis, R. J., \& Evans, M. D. (1987). Causal mediation of change in treatment for depression: Discriminating between nonspecificity and noncausality. Psychological Bulletin, 102(1), 139-149.

Hollon, S. D., \& Ponniah, K. (2010). A review of empirically supported psychological therapies for mood disorders in adults. Depression and Anxiety, 27(10), 891-932.

Hollon, S. D., Thase, M. E., \& Markowitz, J. C. (2002). Treatment and prevention of depression. Psychological Science and Public Interest, 3, 39-77.

Horowitz, L. M., Alden, L. E., Wiggins, J. S., \& Pincus, A. L. (2000). IIP - inventory of 
interpersonal problems manual. San Antonio, TX: The Psychological Corporation. Horowitz, L. M., Rosenberg, S. E., Baer, B. A., Ureño, G., \& Villaseñor, V. S. (1988). Inventory of interpersonal problems: Psychometric properties and clinical applications. Journal of Consulting and Clinical Psychology, 56, 885-892.

Horvath, A. O. (1994). Empirical validation of Bordin's pantheoretical model of the alliance: The working alliance inventory perspective. In A. O. Horvath, \& L. S. Greenberg (Eds.), The working Alliance: Theory, research, and practice (pp. 109-128). New York, NY: Wiley.

Horvath, A. O., Del Re, A. C., Flückiger, C., \& Symonds, D. (2011). Alliance in individual psychotherapy. Psychotherapy, 48(1), 9-16.

Huibers, M. J. H., Cohen, Z. D., Lemmens, L. H. J. M., Arntz, A., Peeters, F. P. M. L., Cuijpers, P., et al. (2015). Predicting optimal outcomes in cognitive therapy of interpersonal psychotherapy for depressed individuals using the personalized advantage index approach. PLoS One, 10(11), e0140771.

Johansson, P., Høglend, P., Ulberg, R., Amlo, S., Marble, A., Bøgwald, K.-P., et al. (2010). The mediating role of insight for long-term improvements in psychodynamic therapy. Journal of Consulting and Clinical Psychology, 78(3), 438-448.

Kazdin, A. E. (2007). Mediators and mechanisms of change in psychotherapy research. Annual Review of Clinical Psychology, 3, 1-27.

Kazdin, A. E. (2009). Understanding how and why psychotherapy leads to change. Psychotherapy Research, 19(4-5), 418-428.

Klerman, G. L., Weissman, M. M., Rounsaville, B. J., \& Chevron, E. S. (1984). Interpersonal psychotherapy for depression. New York, NY: Basis Books.

Kline, R. B. (2005). Principles and practice of structural equation modeling (2nd ed.). New York, NY: Guilford.

Kraemer, H. C., Wilson, G. T., Fairburn, C. G., \& Agras, W. S. (2002). Mediators and moderators of treatment effects in randomized clinical trials. Archives of General Psychiatry, 59, 877-883.

Kuyken, W., Watkins, E., Holden, E., White, K., Taylor, R. S., Byford, S., et al. (2010). How does mindfulness-based cognitive therapy work? Behaviour Research and Therapy, 48, 1105-1112.

Lemmens, L. H. J. M., Arntz, A., Peeters, F. P. M. L., Hollon, S. D., Roefs, A., \& Huibers, M. J. H. (2011). Effectiveness, relapse prevention and mechanisms of change of cognitive therapy vs. interpersonal therapy for depression: Study protocol for a randomised controlled trial. Trials, 12(1), 150-162.

Lemmens, L. H. J. M., Arntz, A., Peeters, F. P. M. L., Hollon, S. D., Roefs, A., \& Huibers, M. J. H. (2015). Clinical effectiveness of cognitive therapy vs. Interpersonal psychotherapy for depression: Results of a randomized controlled trial. Psychological Medicine, 45, 2095-2110.

Lemmens, L. H. J. M., DeRubeis, R. J., Arntz, A., Peeters, F., \& Huibers, M. J. H. (2016). Sudden gains in cognitive therapy and interpersonal psychotherapy for adult depression. Behaviour Research and Therapy, 77, 170-176.

Lemmens, L. H. J. M., Müller, V. N. L. S., Arntz, A., \& Huibers, M. J. H. (2016). Mechanisms of change in psychotherapy for depression: An empirical update and evaluation of research aimed at identifying psychological mediators. Clinical Psychology Review, 50, 95-107.

Longmore, R. J., \& Worrell, M. (2007). Do we need to challenge thoughts in cognitive behavior therapy? Clinical Psychology Review, 27(2), 173-187.

Lorenzo-Luaces, L., German, R. E., \& DeRubeis, R. J. (2014). It's complicated: The relation between cognitive change procedures, cognitive change, and symptom change in cognitive therapy for depression. Clinical Psychological Review, 41, $3-15$.

MacKinnon, D. P. (2008). Introduction to statistical mediation analysis. New York, NY: Taylor \& Francis Group.

MacKinnon, D. P., Fairchild, A. J., \& Fritz, M. S. (2007). Mediation analysis. Annual
Review of Clinical Psychology, 58, 593-614.

Markowitz, J. C., \& Weissman, M. M. (2004). Interpersonal psychotherapy: Principles and applications. World Psychiatry, 3, 136-139.

McArdle, J. J. (2009). Latent variable modeling of differences and changes with longitudinal data. Annual Review of Psychology, 60, 577-605.

Muthén, B. O., \& Asparouhov, T. (2014). Causal effects in mediation modeling: An introduction with applications to latent variables. Structural Equation Modeling: A Multidisciplinary Journal, 22(1), 12-23.

Nolen-Hoeksema, S., \& Morrow, J. (1991). A prospective study of depression and posttraumatic stress symptoms after a natural disaster: The 1989 Loma Prieta earthquake. Journal of Personality and Social Psychology, 61(1), 115-121.

Quilty, L. C., McBride, C., \& Bagby, R. M. (2008). Evidence for the cognitive mediational model of cognitive behavioural therapy for depression. Psychological Medicine, 38, 1531-1541.

Raes, F., Hermans, D., \& Eelen, P. (2003). Kort instrumenteel de nederlandstalige versie van de Ruminative Response Scale (RRS-NL) en de Rumination on Sadness Scale (RSS-NL). Gedragstherapie, 36(2), 97-104.

Renner, F., Jarret, R. B., Vittengl, J. R., Barret, M. S., Clark, L. A., \& Thase, M. E. (2012) Interpersonal problems as predictors of therapeutic alliance and symptom improvement in cognitive therapy for depression. Journal of Affective Disorders, 138(3), 458-467.

Strunk, D. R., Brotman, M. A., \& DeRubeis, R. J. (2010). The process of change in cognitive therapy for depression: Predictors of early inter-session symptom gains. Behaviour Research and Therapy, 48(7), 599-606.

Tafarodi, R. W., \& Swann, W. B. (2001). Two-dimensional self-esteem: Theory and measurement. Personality and Individual Differences, 31(5), 653-673.

Tichenor, V., \& Hill, C. E. (1989). A comparison of six measures of working alliance. Psychotherapy: Theory, Research, Practice, Training, 26(2), 195-199.

Toth, S. L., Rogosch, F. A., Oshri, A., Gravener-Davis, J., Sturm, R., \& MorganLópez, A. A. (2013). The efficacy of interpersonal psychotherapy for depression among economically disadvantaged mothers. Development and Psychopathology, 25, 1065-1078.

Tracey, T. J., \& Kokotovic, A. M. (1989). Factor structure of the working alliance inventory. Psychological Assessment: A Journal of Consulting and Clinical Psychology, 1(3), 207-210.

Vandromme, H., Hermans, D., Spruyt, A., \& Eelen, P. (2007). Dutch translation of the self-liking/self-competence scale - revised: A confirmatory factor analysis of the two-factor structure. Personality and Individual Differences, 42(1), 157-167.

Warmerdam, L., van Straten, A., Jongsma, J., Twisk, J., \& Cuijpers, P. (2010). Online cognitive behavioral therapy and problem-solving therapy for depressive symptoms: Exploring mechanisms of change. Journal of Behavior Therapy and Experimental Psychiatry, 41, 64-70.

Webb, C. A., DeRubeis, R. J., Amsterdam, J. D., Shelton, R. C., Hollon, S. D., \& Dimidjian, S. (2011). Two aspects of the therapeutic alliance: Differential relations with depressive symptom change. Journal of Consulting and Clinical Psychology, 79(3), 279-283.

Weissman, A. N., \& Beck, A. T. (1978). Development and validation of the dysfunctional attitude scale; paper presented at the annual meeting of the association for the advancement of behavior therapy.

Whisman, M. A. (1993). Mediators and moderators of change in cognitive therapy of depression. Psychological Bulletin, 114, 248-265.

Zuroff, D. C., \& Blatt, S. J. (2006). The therapeutic relationship in the brief treatment of depression: Contributions to clinical improvement and enhanced adaptive capacities. Journal of Consulting and Clinical Psychology, 74, 130-140. 